\title{
Long Time Scales and Black Holes
}

\section{Eliezer Rabinovici*}

Racah Institute of Physics, The Hebrew University, Jerusalem 91904, Israel

E-mail: eliezer@vms.huji.ac.il

\section{José L. F. Barbón ${ }^{\dagger}$}

Department of Physics. Theory Division, CERN. CH 1211 Geneva 23

E-mail: barbon@cern.ch

\begin{abstract}
We discuss the various scales determining the temporal behaviour of correlation functions in the presence of eternal black holes. We point out the origins of the failure of the semiclassical gravity approximation to respect a unitarity-based bound suggested by Maldacena. We find that the presence of a subleading (in the large $\mathrm{N}$ approximation involved) master field does restore the compliance with one bound but additional configurations are needed to explain the more detailed expected time dependence of the Poincare recurrences and their magnitude.
\end{abstract}

\section{Introduction}

Hawking's semiclassical analysis of black hole evaporation suggests that most of the information contained in initial scattering states is shielded behind the event horizon, never to return back to the asymptotic region far from the evaporating black hole [目. In this picture, the singularity is capable of absorbing all the infalling information, which is then destroyed or transmitted to other geometrical realms, depending on one's hypotheses about the microphysics of the singularity. From the point of view of measurements on the Hawking radiation, the evaporation is not described by a unitary S-matrix. Rather, quantum coherence is violated and the linear evolution in Hilbert space takes pure states into mixed states. Still, probability is conserved, since density matrices $\rho$ remain Hermitian, $\rho^{\dagger}=\rho$, positive, $\rho>0$ and normalized, $\operatorname{Tr} \rho=1$ under time evolution.

The AdS/CFT correspondence [2] is not consistent with this picture. In this construction, quantum gravity in a $(d+1)$-dimensional asymptotically Anti-de Sitter spacetime (AdS) of curvature radius $R$ is defined in terms of a conformal field theory (CFT) on a

\footnotetext{
${ }^{*}$ Speaker.

${ }^{\dagger}$ On leave from Departamento de Física de Partículas da Universidade de Santiago de Compostela (Spain)
} 
spatial sphere $\mathbf{S}^{d-1}$ of radius $R$. The effective expansion parameter in the gravity side $1 / N^{2} \sim G_{\mathrm{N}} / R^{d-1}$, maps to an appropriate large $N$ limit of the CFT. For example, for two-dimensional CFT's $N^{2}$ is the central charge. When the CFT is a gauge theory, the AdS side is a string theory, $N$ is the rank of the gauge group, and the string perturbative expansion in powers of $g_{s} \sim 1 / N$ is identified with 't Hooft's $1 / N$ expansion in the gauge theory side.

According to this definition, the formation and evaporation of small black holes with Schwarschild radius $R_{S} \ll R$, should be described by a unitary process in terms of the CFT Hamiltonian. Thus, there is no room for violations of coherence as a matter of principle. Unfortunately, the CFT states corresponding to small black holes are hard to describe, and it remains a challenge to put the finger on the precise error in Hawking's semiclassical analysis in that case.

For large AdS black holes with Schwarschild radius $R_{S} \gg R$ one may attempt to rise to the challenge, since they are thermodynamically stable and can exist in equilibrium at fixed (high) temperatures $1 / \beta \gg 1 / R$. Indeed, the corresponding Bekenstein-Hawking entropy scales like that of $N^{2}$ conformal degrees of freedom at high energy,

$$
S \sim \sqrt{N}(E R)^{\frac{d-1}{d}} \sim N^{2}(R / \beta)^{d-1} .
$$

Therefore, large AdS black holes with inverse Hawking temperature $\beta \ll R$ describe the leading approximation to the thermodynamical functions of the canonical CFT state

$$
\rho_{\beta}=\frac{e^{-\beta H}}{Z(\beta)}, \quad Z(\beta)=\operatorname{Tr} \exp (-\beta H) .
$$

This suggests that we can test the semiclassical unitarity argument by careful analysis of slight departures from equilibrium, rather than studying a complete evaporation instability in the vacuum. Ref. 3 proposes to look at the very long time structure of correlators of the form

$$
G(t)=\operatorname{Tr}[\rho A(t) A(0)],
$$

for appropriate Hermitian operators $A$. In the semiclassical approximation, one expects these correlators to decay as $\exp (-\Gamma t)$ with $\Gamma \sim \beta^{-1}$. However, because the CFT lives in finite volume, the spectrum is actually discrete (c.f. Fig 1), and the correlator must show nontrivial long time structure in the form of Poincaré recurrences (see [4]). This result, which is straightforward from the boundary theory point of view, has far reaching consequences as far as the bulk physics is concerned.

Hence, the failure of $G(t)$ to vanish as $t \rightarrow \infty$ can be used as a criterion for unitarity preservation beyond the semiclassical approximation. This argument can be made more explicit by checking the effect of coherence loss on the long-time behaviour of $G(t)$. Using the results of [6] one can simulate the required decoherence by coupling an ordinary quantum mechanical system to a random classical noise. It is then shown in [7] that this random noise forces $G(t)$ to decay exponentially for large $t$, despite having a discrete energy spectrum. This shows that the long-time behaviour of correlators probes the strict quantum coherence of the bounded system. 
At the same time, one would like to identify what kind of systematic corrections to the leading semiclassical approximation are capable of restoring unitarity. A proposal was made in [3] in terms of topology-changing fluctuations of the AdS background. Our purpose here is to investigate these questions and offer an explicit estimate of the instanton effects suggested in [3] (see also [8]). Ultimately, this analysis should provide information about the nature of the black hole singularity.

\section{Long-time Details of Thermal Quasi- equilibrium}

Poincaré recurrences occur in general bounded systems. Classically they follow from the compactness of available phase space, plus the preservation of the phase-space volume in time (Liouville's theorem). Quantum mechanically, they follow from discreteness of the energy spectrum (characteristic of spatially bounded systems) and unitarity, since

$$
G_{\beta}(t)=\frac{1}{Z(\beta)} \sum_{i, j} e^{-\beta E_{i}}\left|A_{i j}\right|^{2} e^{i\left(E_{i}-E_{j}\right) t}
$$

defines a quasiperiodic function of time (we have chosen the canonical density matrix for the initial state). After initial dissipation on a time scale $\Gamma^{-1}$, where $\Gamma$ measures the approximate width of matrix elements of $A$ in the energy basis, the correlator will show $O(1)$ "resurgences" when most of the relevant phases complete a period (c.f. Fig 2). The associated time scale is $t_{H} \equiv 1 /\langle\omega\rangle$, with $\langle\omega\rangle=\left\langle E_{i}-E_{j}\right\rangle$ an average frequency in (2.1). We can estimate $\langle\omega\rangle$ as $\Gamma / \Delta n_{\Gamma}$, where $\Delta n_{\Gamma}$ is the number of energy levels in the relevant band of width $\Gamma$. Introducing the microcanonical entropy in terms of the level-number function as $n(E) \equiv \exp S(E)$, we have

$$
\Delta n_{\Gamma} \approx \int_{\langle E\rangle-\Gamma / 2}^{\langle E\rangle+\Gamma / 2} d E \frac{d n}{d E}=\int_{\langle E\rangle-\Gamma / 2}^{\langle E\rangle+\Gamma / 2} d E \beta(E) e^{S(E)} \approx \Gamma \beta e^{S(\beta)} .
$$

where we have introduced the microcanonical inverse temperature as $\beta(E) \equiv d S / d E$.

From this analysis we obtain an estimate

$$
t_{H} \sim \beta e^{S(\beta)} .
$$

Following [9] we call this the Heisenberg time scale. Poincaré times can be defined in terms of quasiperiodic returns of $G_{\beta}(t)$ with a given a priori accuracy. In a sense, the Heisenberg time is the smallest possible Poincaré time. 


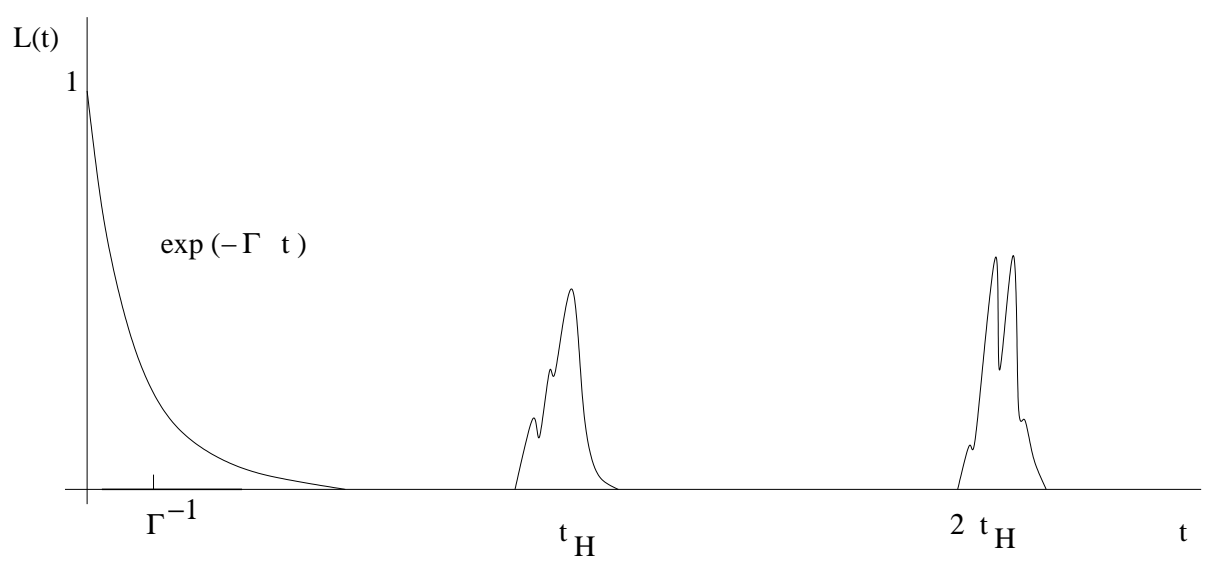

Figure 2: Schematic representation of the very long time behaviour of the normalized time correlator $L(t)$ in bounded systems. The initial decay with lifetime of order $\Gamma^{-1}$ is followed by $O(1)$ "resurgences" after the Heisenberg time $t_{H} \sim \beta \exp (S)$ has elapsed. Poincaré recurrence times can be defined by demanding the resurgences to approach unity with a given a priori accuracy, and scale like a double exponential of the entropy.

A more quantitative criterion can be used by defining a normalized positive correlator, $L(t)$, satisfying $L(0)=1$, and its infinite time average,

$$
L(t) \equiv\left|\frac{G(t)}{G(0)}\right|^{2}, \quad \bar{L} \equiv \lim _{T \rightarrow \infty} \frac{1}{T} \int_{0}^{T} d t L(t) .
$$

The profile of $L(t)$ is sketched in Fig 2. The time average can be estimated by noticing that the graph of $L(t)$ features positive "bumps" of height $\Delta L$ and width $\Gamma$, separated a time $t_{H}$, so that

$$
\bar{L} \sim \frac{\Delta L}{\Gamma t_{H}} .
$$

For the case at hand $\Delta L \sim 1, t_{H} \sim \beta e^{S}$, and we obtain (c.f. [5, 7])

$$
\bar{L} \sim \frac{e^{-S(\beta)}}{\beta \Gamma} .
$$

Since both $\beta$ and $\Gamma$ scale as $N^{0}$ in the large- $N$ limit of the dual CFT, the "recurrence index" $\bar{L} \sim \exp \left(-N^{2}\right)$ scales as a nonperturbative effect in the semiclassical approximation.

Indeed, one finds $\bar{L}=0$ in gravity perturbation theory in the AdS black hole background. The reason is that the relevant eigenfrequencies $\omega$ (the so-called normal modes of the black hole) form a continuous spectrum to all orders in the $1 / N$ expansion. For a static metric of the form

$$
d s^{2}=-g(r) d t^{2}+\frac{d r^{2}}{g(r)}+r^{2} d \Omega_{d-2}^{2},
$$

the normal frequency spectrum follows from the diagonalization of a radial Schrödinger operator

$$
\omega^{2}=-\frac{d^{2}}{d r_{*}^{2}}+V_{\mathrm{eff}}\left(r_{*}\right)
$$


with

$$
V_{\mathrm{eff}}=\frac{d-2}{2} g(r)\left(\frac{g^{\prime}(r)}{r}+\frac{d-4}{2 r^{2}} g(r)\right)+g(r)\left(-\frac{\nabla_{\Omega}^{2}}{r^{2}}+m^{2}\right)
$$

for a scalar field of mass $m$ (analogous potentials can be deduced for higher spin fields). Here we have defined the Regge-Wheeler or "tortoise" coordinate $d r_{*}=d r / g(r)$.
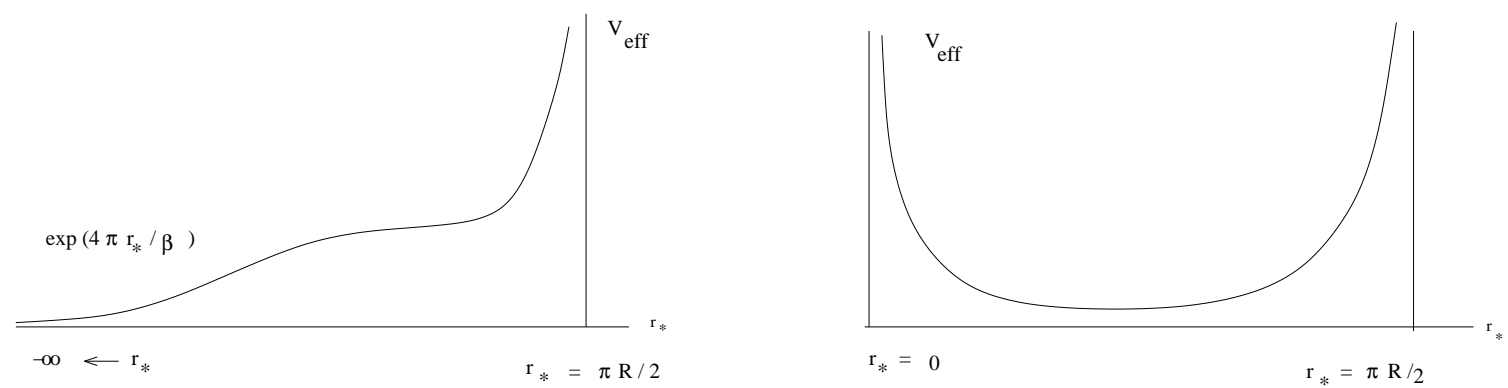

Figure 3: The effective potential determining the semiclassical normal frequency modes in a large AdS black hole background (left). In Regge-Wheeler coordinates the horizon is at $r_{*}=$ $-\infty$, whereas the boundary of $A d S$ is at $r_{*}=\pi R / 2$ (only the region exterior to the horizon appears). There is a universal exponential behaviour in the near-horizon (Rindler) region. The effective one-dimensional Schrödinger problem represents a semi-infinite barrier with a continuous energy spectrum. This contrasts with the analogous effective potential in vacuum AdS with global coordinates (right). The domain of $r_{*}$ is compact and the spectrum of normal modes is discrete with gap of order $1 / R$.

We have shown in Fig. 3 the form of the resulting effective potentials for large AdS black holes, compared with the case of the vacuum AdS manifold. The vacuum AdS manifold, corresponding to the choice $g(r)=1+r^{2} / R^{2}$ in (2.7), behaves like a finite cavity, as expected. The distinguishing feature of the black-hole horizon is a a non-degenerate zero, $g\left(r_{0}\right)=0$, which induces the universal scaling

$$
V_{\mathrm{eff}}\left(r_{*}\right) \propto \exp \left(4 \pi r_{*} / \beta\right) \quad \text { as } \quad r_{*} \rightarrow-\infty,
$$

with $1 / \beta=g^{\prime}\left(r_{0}\right) / 4 \pi$ the Hawking temperature and the horizon $r=r_{0}$ appearing at $r_{*}=-\infty$. The spectrum is thus discrete in pure AdS, and continuous in the AdS black hole. Physically, this just reflects the fact that the horizon is an infinite redshift surface, so that we can store an arbitrary number of modes with finite total energy, provided they are sufficiently red-shifted by approaching the horizon [10. Since the thermal entropy of perturbative gravity excitations in the vacuum AdS spacetime scales as $S(\beta)_{\operatorname{AdS}} \sim N^{0}$, we see that the perturbative Heisenberg time of the AdS spacetime is of $O(1)$ in the large- $N$ limit, leading to $\bar{L}_{\mathrm{AdS}}=O(1)$. On the other hand, we have $\bar{L}_{\mathrm{bh}}=0$ in this approximation.

\section{Topological Diversity and Unitarity}

It is instructive to understand these perturbative results in the Euclidean formalism, obtained by $t=-i \tau$ in (2.7), followed by an identification $\tau \equiv \tau+\beta$. The resulting metric for the vacuum AdS spacetime has a non-contractible $\mathbf{S}^{1}$ given by the $\tau$ compact direction. 
We call $Y$ this Euclidean manifold. On the other hand, the black hole spacetime with $g\left(r_{0}\right)=0$ has different topology, since the thermal $\mathbf{S}^{1}$ shrinks to zero size at $r=r_{0}$. The choice $1 / \beta=g^{\prime}\left(r_{0}\right) / 4 \pi$ ensures smoothness at $r=r_{0}$. We call this Euclidean black hole manifold $X$.

The real-time correlation functions in the black hole background, $G(t)_{X}$, follow by analytic continuation from their Euclidean counterparts. Since $X$ is a completely smooth manifold in the $1 / N$ expansion, so is the Euclidean correlator $G(i t)_{X}$ for $t \neq 0$. The continuous spectrum arising in the spectral decomposition of $G(t)_{X}$ is a consequence of the contractible topology of $X$, since the Hamiltonian folliation by $\tau=$ constant surfaces is singular at $r=r_{0}$.
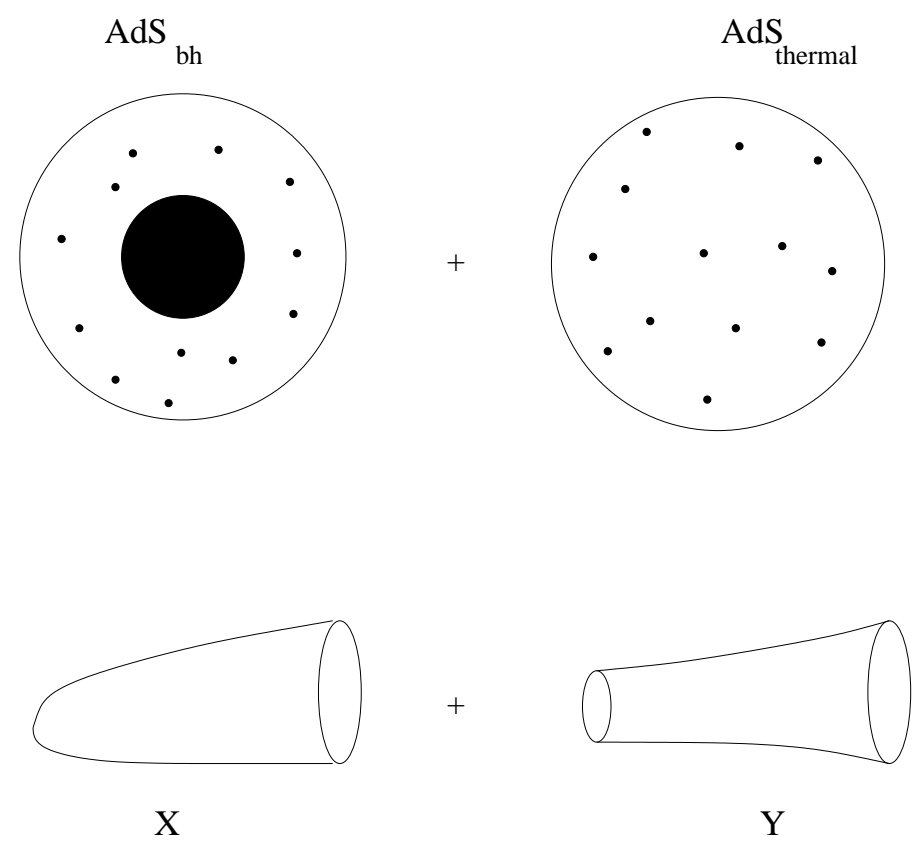

Figure 4: Summing over large-scale fluctuations of the thermal ensemble in which a black hole spontaneously turns into radiation (and viceversa) is represented in the Euclidean formalism as the coherent sum of thermal saddle points of different topology. The "cigar-like" geometry $X$ represents the black-hole master field (in the CFT language) and the cylindrical topology $Y$ represents the thermal gas of particles.

Therefore, it seems that improving on the semiclassical prediction for $\bar{L}$ requires some sort of topology-change process. The proposal of [3] is precisely that: instead of evaluating the semiclassical correlators on $X$, one should sum coherently the contribution of $X$ and $Y$. Normally one neglects the contribution of $Y$ on a quantitative basis (at high temperatures $R \gg \beta)$. However, here the contribution of $X$ to $\bar{L}$ vanishes and one is forced to consider the first correction. Since $Y$ has a discrete spectrum in perturbation theory, the net result for $\bar{L}$ should be nonzero in this approximation. Physically, this superposition of Euclidean saddle points (or master fields, in the language of the CFT) corresponds to large-scale fluctuations in which the AdS black hole is converted into a graviton gas at the same temperature and viceversa. 
A more detailed estimate of this "instanton" approximation to $\bar{L}$ yields (c.f. [7])

$$
\bar{L}_{\mathrm{inst}} \approx C e^{-2 \Delta I}
$$

where $C=O\left(N^{0}\right), \Delta I=I_{Y}-I_{X}$ and $I=-\log Z(\beta)$, calculated in the classical gravity approximation. Since $I_{Y} \sim-N^{0}$ and $I_{X} \sim-N^{2}$, the exponential suppression factor is of order $\exp \left(-2\left|I_{X}\right|\right) \sim \exp \left(-N^{2}\right)$, reproducing the expected scaling (2.6), at least in order of magnitude (however, in general $S_{X} \neq-2\left|I_{X}\right|$, even at high temperature).

However, the apparent success of (3.1) turns out to be somewhat coincidental. If we consider the full time profile of $L(t)$ rather than the infinite time average, we find

$$
L(t)_{\mathrm{inst}} \approx L(t)_{X}+C e^{-2 \Delta I} L(t)_{Y}
$$

The resulting structure is shown in Figs. 5 and 6 . The instanton approximation to the normalized correlator features the normal dissipation with lifetime $\Gamma^{-1} \sim \beta$ coming from the contribution of $X$. However, the resurgences are controlled by $L(t)_{Y}$, damped by a factor $\exp (-2 \Delta I) \sim \exp \left(-N^{2}\right)$, and separated a time $t_{H}(Y) \sim N^{0}$.

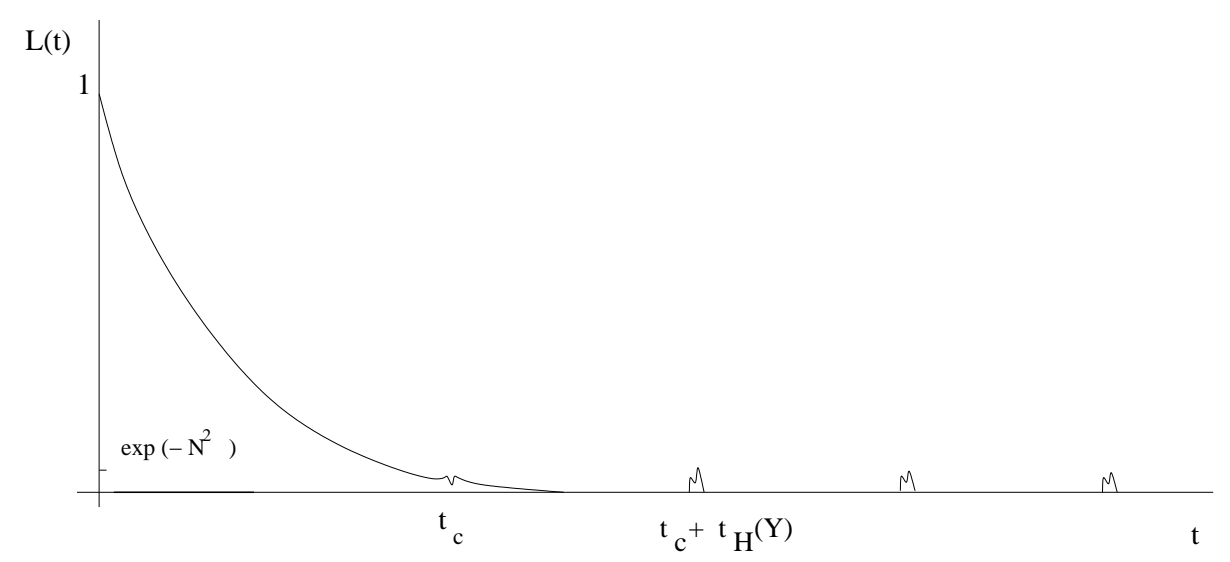

Figure 5: The instanton approximation to the correlator $L(t)_{\text {inst }}$ features the expected exponential decay $\exp (-\Gamma t)$ induced by the contribution of the $X$-manifold, whereas the resurgences are entirely due to the interference with the $Y$-manifold, leading to small bumps of order $\exp (-2 \Delta I) \sim \exp \left(-N^{2}\right)$, separated a time $t_{H}(Y) \sim N^{0}$. These bumps are noticeable against the background of the $X$-manifold after a time $t_{c} \sim \Delta I / \Gamma$.

Hence, the very long time behaviour as shown in Fig. 6 is very different from the expected one, although the infinite time average comes out right in order of magnitude:

$$
\bar{L} \sim \frac{\Delta L}{\Gamma t_{H}} \sim \frac{e^{-N^{2}}}{\Gamma \cdot \beta} \sim \frac{1}{\Gamma \cdot \beta e^{N^{2}}}
$$

We can also find the time scale $t_{c}$ for which the large-scale instantons considered here are quantitatively important on the graph of $L(t)$. This is shown in Fig. 5 and yields $t_{c} \sim \Delta I / \Gamma \sim N^{2}$. 


\section{Conclusions}

The study of very long time features of correlators in black hole backgrounds is a potentially important approach towards unraveling the mysteries of black hole evaporation and the associated physics at the spacelike singularity. We have seen that large scale topology-changing fluctuations proposed in [3] begin to restore some of the fine structure required by unitarity, but fall short at the quantitative level. Presumably the appropriate instantons occur on microscopic scales and involve stringy dynamics.

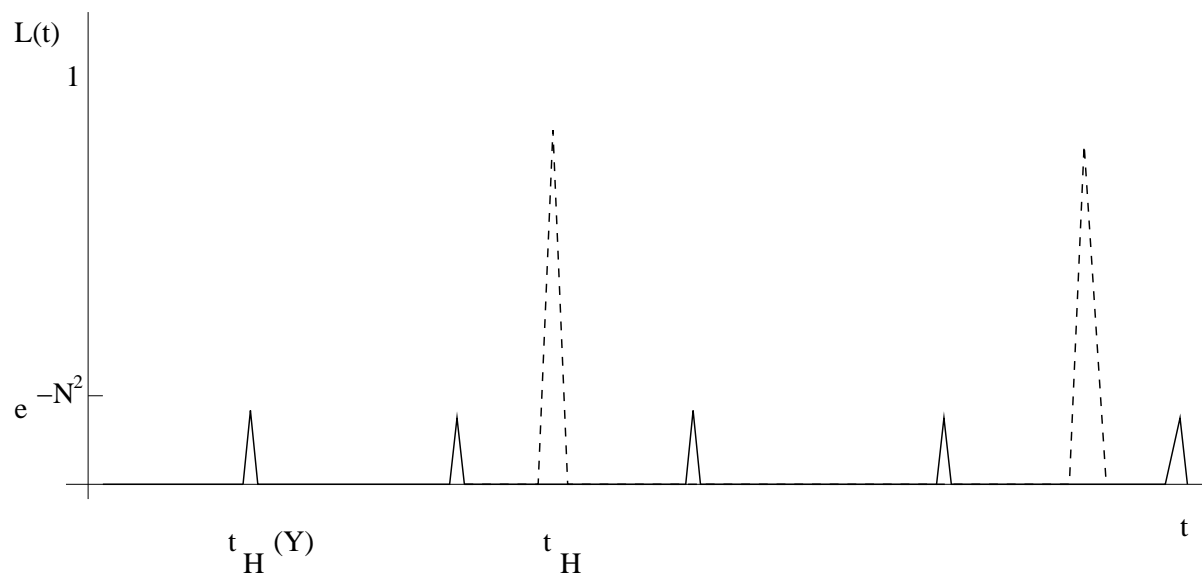

Figure 6: Schematic representation of the very long time behaviour of $L(t)_{\text {inst }}$ (dark line) compared to the expected pattern for the exact quantity $L(t)$. The resurgences of $L(t)_{\text {inst }}$ occur with periods of order $t_{H}(Y)=O\left(N^{0}\right)$ and have amplitude of order $\exp \left(-N^{2}\right) \ll 1$. The expectations for the exact $C F T$, in the dashed line, are $O(1)$ resurgences with a much larger period $t_{H} \sim \exp \left(N^{2}\right) \gg t_{H}(Y)$, corresponding to tiny energy spacings of order $\exp \left(-N^{2}\right)$. Despite the gross difference of both profiles, the infinite time average is $O\left(e^{-N^{2}}\right)$ for both of them.

While semiclassical black holes do faithfully reproduce "coarse grained" inclusive properties of the system such as the entropy (c.f. [11]), additional dynamical features of the horizon may be necessary to resolve finer details of the information loss problem. Roughly, one needs a systematic set of corrections that could generate a "stretched horizon" of Planckian thickness [12]. The crudest model of such stretched horizon is the brick-wall model of 't Hooft 10]. In this phenomenological description one replaces the horizon by a reflecting boundary condition at Planck distance $\epsilon \sim \ell_{P}$ from the horizon. This defines a "mutilated" $X_{\epsilon}$ manifold, of cylindrical topology, leading to a discrete spectrum of the right spacing in order of magnitude.

We have also seen that the characteristic time for large topological fluctuations to be important is $t_{c} \sim O\left(N^{2}\right)$ in the semiclassical approximation. In [13] it was argued that semiclassical two-point functions probe the black hole singularity on much shorter characteristic times, thereby justifying the analysis on the single standard black hole manifold. However, we have seen that detailed unitarity is only restored on time scales of order $t_{H} \sim \exp \left(N^{2}\right)$. Thus $t_{c} \ll t_{H}$ and we conclude that such semiclassical analysis of the singularity is bound to be incomplete, as it misses whatever microphysics is responsible for the detailed unitarity restoration in the quantum mechanical time evolution. 
Acknowledgement E. R. would like to thank the KITP at Santa Barbara for hospitality during the completion of this work. The work of J.L.F.B. was partially supported by MCyT and FEDER under grant BFM2002-03881 and the European RTN network HPRNCT-2002-00325. The work of E.R. is supported in part by the National Science Foundation under Grant No. PHY99-07949, Miller Foundation, the BSF-American Israeli Bi-National Science Foundation, The Israel Science Foundation-Centers of Excellence Program, The German-Israel Bi-National Science Foundation and the European RTN network HPRNCT-2000-00122.

\section{References}

[1] S.W. Hawking, Breakdown of predictability in gravitational collapse, Phys. Rev. D14 (1976) 2460.

[2] J. Maldacena, The large $N$ limit of superconformal field theories and supergravity, Adv. Theor. Math. Phys. 2 (1998) 231 [hep-th/9711200]. S.S. Gubser, I.R. Klebanov and A.M. Polyakov, Gauge theory correlators from noncritical string theory, Phys. Lett. B428 (1998) 105 [hep-th/9802109]. E. Witten, Anti-de Sitter space and holography, Adv. Theor. Math. Phys. 2 (1998) 253 [hep-th/9802150].

[3] J. Maldacena, Eternal black holes in anti-de Sitter, J. High Energy Phys. 0304 (2003) 021 [hep-th/0106112].

[4] L Susskind, Twenty years of debate with Stephen, Contribution to Stephen Hawking's 60th birthday celebration. [hep-th/0204027]. L. Dyson, J. Lindesay and L. Susskind, Is there really a de Sitter/CFT duality, J. High Energy Phys. 0208 (2002) 045 [hep-th/0202163]. N. Goheer, M. Kleban and L. Susskind, The trouble with de Sitter space, J. High Energy Phys. 0307 (2003) 056 [hep-th/0212209].

[5] L. Dyson, M. Kleban and L. Susskind, Disturbing implications of a cosmological constant, J. High Energy Phys. 0210 (2002) 011 [hep-th/0208013].

[6] T. Banks, L. Susskind and M. E. Peskin, Difficulties for the evolution of pure states into mixed states, Nucl. Phys. B244 (1984) 125.

[7] J.L.F. Barbón and E. Rabinovici, Very long time scales and black hole thermal equilibrium, J. High Energy Phys. 0311 (2003) 047 [hep-th/0308063].

[8] D. Birmingham, I. Sachs, S. N. Solodukhin, Relaxation in conformal field theory, Hawking-Page phase transition and quasinormal/normal modes, Phys. Rev. D67 (2003) 104026 [hep-th/0212308]. I. Sachs, Quasinormal modes, [hep-th/0312287.]

[9] M. Srednicki, The approach to thermal equilibrium in quantized chaotic systems, [cond-mat/9809360].

[10] G. 't Hooft, On the quantum structure of a black hole, Nucl. Phys. B256 (1985) 727.

[11] G.W. Gibbons and S.W. Hawking, Action integrals and partition functions in quantum gravity, Phys. Rev. D15 (1977) 2752.

[12] K.S. Thorne, R.H Price and D.A. McDonald, Black Holes: The Membrane Paradigm. Yale University Press 1986. L. Susskind, L. Thorlacius and J. Uglum, The stretched horizon and black hole complementarity, Phys. Rev. D48 (1993) 3743 [hep-th/9306069]. 
[13] P. Kraus, H. Ooguri and S. Shenker, Inside the horizon with AdS/CFT, Phys. Rev. D67 (2003) 124022 [hep-th/0212277]. L. Fidkowski, V. Hubeny, M. Kleban and S. Shenker, The black hole singularity in AdS/CFT, [hep-th/0306170]. 